\section{A CLINICAL STUDY}

OF A

\section{CASE OF CPST OF THE CEREBELLUM :}

\section{Weakness of Spinal Muscles : Death from Fatlure of Respiration.}

By J. HUGHLINGS JACKSON, M.D., F.R.S.,

Physician to the London Hospital, and to the National Hospital for the Paralysed and Epileptic;

AND

J. S. RISIEN RUSSELL, M.D., M.R.C.P., Assistant Physician to the Metropolitan Hospital

OUR main object in recording this case is to emphasise a point in the symptomatology of cerebellar disease to which iittle attention has been paid. There is evidence of paresis of the trunk muscles, a direct result, we believe, of the structural damage to the cerebellum produced by the cyst. We record the case also as it exemplifies a mode of dying in cases of intracranial tumour first pointed out by the late Dr. Hilton Fagge-death by failure of respiration.

It has long been maintained by one of us (J. H. J.) that destructive lesions of the cerebellum, of its middle lobe at least, produce true motor paresis or paralysis, and that the trunk muscles are first and most affected, those of the inferior extremities next in order and severity, and those of the superior extremities last and least. Thus the degree and order of involvement of the different parts mentioned is the reverse of that which obtains in a destructive lesion of the cerebrum attended by paralysis, for in most cases of hemiplegia the arm suffers most, the leg less, and the trunk least. The recent experimental investigations of one of us (J. S. R. R.) appear to support these views on motor representation by the cerebellum. The results of these experiments in dogs and monkeys seem to prove the existence of motor paresis as one factor in the causation of the inability on the part of the animal to walk or stand after ablation of parts or the whole of the cerebellum; and it was evident that in the dogs the posterior extremities suffered far more than the anterior. The condition of the trunk muscles could not be so well judged of in these animals, but in monkeys, after extirpation of the whole of the cerebellum, the paresis of the back muscles was so great that the animal was unable to sit up.

Before going further, we must point out that paresis of the spinal muscles in cases of disease of the cerebellum was pointed out long ago by Niemeyer. He wrote : "In tumours of the cerebellum, as we have previously shown, hemiplegia is not constant, and when present is not pure, but extends to the other side of the body to a less extent, especially affecting the muscles of the spinal column. Paresis of the muscles inducing bending, erection, and lateral movements of the spinal column, first shows itself by a peculiar form of dizziness, which we have already fully described. and referred to vibrations of the spine in walking and similar acts ; subsequently, it often evinces itself by an utter inability of the body to maintain itself, so in the sitting position the patients collapse and cannot move the body, unless held by both shoulders."

We are indebted to Dr. J. B. Bird for the careful notes of the clinical history of the following case, and for his assistance in the investigation of the points of special interest; to Dr. James Taylor, then fellow house-physician of one of us (J. S. R. R.), for similar valuable aid; and to Dr. W. S. Colman for the record of the post-mortem observations made by him. A. S,, aged 30 years, was admitted into the National Hospital for the symptoms believed to point to

I A Text-Book of Practical Medicine. By Dr. Felix von Niemeyer. Translated from the Eighth German Edition by George H. Humphreys, M.D., and Charles E. Hackley, M.D. Vol. ii, p. 244. 1876.
History. - Threa years bc fore admission he suffered from headache vomiting, staggering with a 1endeucy to fall backwards, and from "giddy ject to attacks in wher of whi inh he did not remember. He was also sub not; these and all the other symptrul whether he lost consciousness or the headache, which per'sisted. (This symptom had been in existence of four or flve years, but the patient was in the early part of the period constipated, and the headache used to be relieved when his bowels acted). A year later he was thrown down, struck tlie back of his head, and was quite well next day. Six inonths after this he was diving and struek was vertex of his head on a stone which rhis he was diving, and struck the an hour. He vomited off and on for five weeks after this, and then was well enough to resume his duties as a police constable.

In January, 1889, that is a year before he came under our observation he began to vomit nearly every morning on first getting up, before food was taken, and also during the day, especially after meals. On looking up, or on looking suddenly round to the right or left, he became giddy. up, or on looking suddenly round to the right or left, he became giddy. if he closed his eyes. If he turned abruptly in bed at night he felt giddy if and stupid, and took some time to be sure of his position. When giddy pain from which he suffered was first in the forehead, then in the pain from which he suffered was first in the forehead, then in the occipital region; latterly the vertex and the centre of his head was its when all the symptoms became more marked, and had been increasing when all the symptoms became more marked, and had been increasing ever since, especially during the last month before his admission into hospital. Jerkings of both hands and feet were noted six weeks before was not affected. Ten days before admission sight began to be impaired, and three days later he could not, he told us, read; but he could do so at the time when we examined him. During the first three months of 1889 he was troubled by a subjective sensation of ringing in the ears. The patient had previously had good health; no history of syphilis could be elicited. There was no eviden
nant or tuberculous disease.

State on Admission.-The patient was a tall well-developed man, but looked pale, worn, and drowsy. It was evidently a great effort for him to speak and think.

Head. - The circumference of his head was 22 inches. Percussion of the skull elicited general tenderness, no one point being more tender than another. The sense of smell was less acute on the left side than on the right. Vision was ${ }_{6}^{6}$ on the right, and $\frac{e}{5}$ on the left; there was no hemianopia ; there was intense double optic neuritis. Neither ptosis nor strabismus, existed, but on lateral deviation the globes moved in a "punctuated" manner, and there were occasionally nystagmoid jerks. The pupils were 5 millimetres in diameter, and equal; they reacted normally to light and on accommodation. The motor and sensory divisions of the fifth cranial nerves were intact. The only evidence of asymmetry of the face consisted in a trifling drooping of the right angle of the mouth, evident, also, on showing the teeth ; but on smiling and closing the eyes tightly, the facial movements on the two sides were equal. The power to raise the eyebrows and to frown was lost, and there were no transverse
lines across the forehead. The ticking of a watch was heard 6 inches off lines across the forehead. The tirking of a watch was heard 6 inches off each ear. The tongue turned slightly to the right when protruded, and
was tremulous. The uvula was deflected in the slightest degree to the was tremulous. The uvula was deflected in the slightest degree to the
left; but the movements of the palate were equal on the two sides. The palatal reflex was present, and equal on the two sides. There was no dysphagia.

Neck.-All forms of sensibility were normal. Active and passive movements of the head on the trunk were restricted in all directions, as every change in the position of the head increased the pain in it, so that the patient was afraid to move it or allow it to be moved. There was no
rigidity of the muscles, though there was occasionally a subjective feel-
ing of stiffness in them.

Superior Extremities. - Sensibility of all kinds was intact in these extremities, and there were no subjective sensations. He was righthanded, and the right grasp registered 120 on the dynamometer scale the left 100. Muscular sense was normal, as tested by position and weights; but in attempting to touch the nose when his eyes were closed, the left hand was slightly at fault. There was no rigidity; the tendon reflexes were well marked, the left slightly greater than the right

Trunk.-Every variety of sensibility was normal. The abdominal superficial reflexes were present and equal on the two sides. There was slight bint distinct weakness of the back muscles, as evidenced by the existence of "saddle back"' when the patient stood with his feet together. Further evidence of weakness of the back muscles was obtained by placing the patient flat on his abdomen, and then making him dorsiflex his spine by raising his head and trunk as far as possible off the ground without the aid of his arms. The vertical distance between the manubrium sterni and the ground was then measured, as was the distance between the manubrium and the furthest point to which the observer's hand could be pushed under the patient, without undue force, between the trunk and the floor. The former of these measurements was 5 inches, and the latter $6 \frac{1}{4}$ inches; whereas the average in three normal subjects was 8 inches in the former and $10^{3}$ inches in the latter. That the abdomina muscles as flexors of the spine shared in this weakness was evident from the fact that when placed flat on his back with his arms folded across his clest, the patient was unable to sit up, even when he was aided by his legs being pressed close to the ground, a manceuvre which greatly facililegs being pressed close to the ground, a mancuvre which greatly facili-
tates the act of rising into the sitting posture. Sitting up can usually be accomplished by normal subjects in circumstances similar to those be acwhich the patient was tested, and without aid by pressing on these unde which the patient was tested, and without aid by pressing on the legs. Inferior Extremities. - There was no sensory disturbance, either sub-
jective or objective. The plantar and cremasteric reflexes were less marked on the left than they were on the right. The gait was reeling marked on the left than they were on the right. The gait was reeling, but there was no waddling. As the patient lay in bed, all movements of
these extremities were freely carried out, but the power on the right side

2 We regret that there is no description in the notes of the characters of the "saddle back" (lordosis), tut merely the statement that "ther is distinct saddle back when he stands with his feet tcgether."

[1730] 
was slightly less than on the left. There was no rigidity, but the kneejerks were increased, the right more so than the left, and there was ankle clonus on the right side, while there was only a tendency to it on the left. Muscular seuse was normal as far as position was concerned, and only slightly defective as regards the directing of movements as the patient lay in bed. When he reeled, he tended to fall to either side or backward steadily when his eyes were closed.

Rectum.-Constipation was of old standing. There was no loss of control over the sphincter ani.

Bladder.-For six weeks he had only been able to pass water when in the erect posture, and he did so infrequeutl often passing a whole day without doing so. There was no loss of coutrol over the sphincter.

Sexual Power.-There was no abatement of virile power, and he sexual Power.-There was no abatement of

The circulatory, respiratory, alimentary systems, and the urinary
suject to pliapism or to seminal emissions. system (except as just stated), presented no abnormal features until the system (except as just stated), presented no abnorm
dying by respiratory failure, which we now describe.

On February 12th the patient had severe headache during the day, with occasional vomiting, and at 6 P.M. he suddenly lost consciousness, and his breathing ceased, though his pulse continued fairly good. Artificial respiration was commenced at once, and was continued until 2.30 nex morning, when the heart stopped beating. The pulse kept up well for some hours, but then gradually became weaker. During the eight hours and a-half that artificial respitation was continued there was no sign of natural breathing.

Necropsy.-The post-mortem examination revealed a cystic condition of the cerebellum. On cutting through the inferior velum and raising the medulla the fourth ventricle was seen to be widened; its floor was normal, but its roof was formed by the thin transparent floor of a cyst. The posterior wall of the cyst was gelatinous and uneven, possibly indicative of tumour, while the anterior was smooth. The cyst also excavated the lateral lobes, especially the left. The medulla was flattened, as were the cerebral convolutions, and the lateral ventricles of the cerebrum were dilated.

RemaRKs.

This case presents many features of interest which might be commented on, but we shall confine our attention to the condition of the trunk muscles, and to certain general considerations with regard to cerebellar movements, and to the mode of dying.

We submit that the evidence of paresis of the trunk muscles in this case is fairly conclusive. "Saddle back" alone is strong evidence of weakness of the back muscles (extensors and flexors), and, as we have said, this phenomenon was well marked in the case under discussion. Further evidence of the weakness of these muscles was obtained from the investigations into the ability of the patient when lying on his face to raise the trunk from the ground, without the aid of the arms, that is, by the unaided action of the back muscles. It was clear that the patient's power to do this was considerably less than that of normal subjects on whom control experiments were performed. That the abdominal muscles, flexors of the spine, shared in the defect was evidenced by the fact that when placed flat on his back the patient was unable to sit up without the aid of his arms-a feat which is easily accomplished by normal subjects without that aid.

We are of opinion that this condition of paresis of the trunk muscles was a direct result of the destruction of cerebellar tissue, and not a secondary effect resulting from pressure by the cyst on the pyramids. With so much paresis of the trunk muscles, it would be reasonable for us to expect to find marked loss of power in the limbs, had pressure on the pyramids been responsible for the phenomenon, since the extremities always suffer more. than the trunk when the paresis is the result of cutting off of the cerebral influence from the muscles. The fact that so much weakness of the trunk muscles existed when there was so little affection of the muscles of the extremities makes us attribute the weakness of the trunk muscles to cerebellar paralysis rather than to any secondary effect of pressure on the pyramids. We admit, however, that there was great pressure; the patient's hebetude, although it might have been owing to increase of fluid in the lateral ventricles, evidenced this. Moreover, the rapid failure of respiration causing death may have been owing to pressure on the medulla oblongata.

That paresis of the trunk muscles is an early feature of such lesions of the cerebellum has long been insisted by one of us (J. H.J.). The cerebellar reel, the so-called locomotor incoordination, is to be accounted for on the view that the erratic movements of the legs are attempts on their part to "run after" and "prop up" the trunk in its immoderate inclinings resulting from the weakness of the spinal muscles.

With regard to certain of our observations, it may be asserted that there is no mechanism whereby what we have called the cerebellar paralysis can be produced. It has been said that no motor connections between the cerebellum and the spinal cord have been discovered. Recently, however, Marchi has described descending degeneration after lesion, of the cerebellum in dogs and monkeys. ${ }^{3}$ He finds that after ablation of one lateral half of the cerebellum there is a tract of degenerated fibres which occupies the periphery of the antero-lateral region of the spinal cord, on the side of the lesion chiefly, and also of part of the pyramidal tract, and of the anterior part of the direct cerebellar tract, on the side of the lesion. This observer also finds degenerated fibres in the anterior roots. One of us (J. S. R. R.) found degenerated fibres in the cord occupying mainly the antero-lateral region, on the side of the lesion, after ablation of one lateral half of the cerebellum in the dog; whilst in another series of experiments in dogs and monkeys he has found no such degenerated fibres in the cord. At present no satisfactory explanation of this discrepancy has been found. A paper has recently been read before the Royal Society by Dr. Ferrier and Dr. Aldren Turner; they were unable to confirm Marchi's statements as to the existence of a direct efferent cerebellar tract in the spinal cord, or of degeneration in the anterior nerve roots after extirpation of the cerebellum in the monkey. In one case they obtained degeneration in the anterior column, and in another in the lateral column of the cord; but Deiters' nucleus was injured in the first case, and the tegment of the pons, involving the nucleus of the lateral fillet, in the other.

Hence we admit that the facts as to degeneration "descending" from the cerebellum are discrepant; we can thus only rely for support of the hypothesis as to motor representation by the cerebellum, on such clinical facts as we have mentioned and on the extirpation experiments on the cerebellum of dogs and monkeys (J.S. R. R.). We have pointed out that what we believe to be cerebellar paralysis is by some observers accounted for by pressure by growths in the cerebellum on the pyramidal tract; if these are right, then the paralysis is cerebral, not cerebellar as we have suggested.

In this case the failure of respiration will, we suppose, by most be put down to pressure on the medulla injuriously affecting the respiratory centre. It is certainly the most obvious explanation. We have, however, to consider the rapid setting in of the respiratory failure, and also the comparative intactness of the other bulbar centres, those for circulation, for example. For, as we pointed out, the heart continued to beat for eight and a-half hours in the case of A. S. after failure of respiration. One of us (J. H. J.), adopting a principle first stated by Buzzard, has suggested that failure of respiration in cases of intracranial tumour may be owing to changes induced in the respiratory centre, changes akin to those of optic neuritis. Here, again, is the difficulty of the rapid setting in of the respiratory failure ; an analogy may be suggested-that optic neuritis may exist for a long time with good vision, and then sight may rapidly fail. We do not, however, in this paper discuss that hypothesis. We wish to draw particular attention to some researches of great importance by Mr. Walter Spencer and Mr. Victor Horsley ${ }^{4}$ bearing closely on the matter in question. These researches are important in many other clinical regards. Mr. Spencer and $\mathrm{Mr}$. Horsley record the results of experiments on dogs and on a few monkeys. Intracranial pressure was artificially raised by the insertion of a thin-walled, easily distensible india-rubber bag; the bag was inserted at various points, from the supra-orbital region in front to the cerebellar region behind. By inflating the bag they produced well-marked effects on circulation and respiration. They write (p. 211): "When pressure is applied to the cerebral hemisphere. especially above and in front, we have such displacement of the encephalon that, in spite of the support given by the tentorium (which, it is to be remembered, is bony for the mos! " rt in the carnivora), the cerebellum is driven through the if : " men magnum."

They show that the effects on circulation and respiration they produced are not owing to excitations from the brain immediately pressed on, for they obtained the same results when the mesencephalon was divided and thus when the ex3 Marchi, Riv. Sper. d. Fren. e Med. Leg. Anno XII, 1886, F. I; A. XIII, 1887, F. IV. A. XViI 1891 4 Trans. Roy. Soc. B., 1891, p. 201. 
citations spoken of, if there were any, could not reach the medulla. The following extract (p. 244) will give some idea of the value of their researches: "If depressor influences were active and had not been impaired in any way then the slowing of the respiration was accompanied by slowing of the heart and a fall of blood pressure. When the respiration was arrested the heart was greatly slowed and then stopped. But if the depressor influence had been lost the heart was only slowed a little on the arrest of respiration and there was no fall of blood pressure nor arrest of the heart and the slight slowing was altogether lost when the heart was acting very quickly consequent upon paralysis of the cardio-inhibitory apparatus. The blood pressure affected the heart rate apart from any cardio-inhibitory effect, for after division of the vagi the heart became quickened as the blond pressure rose and slowed as it fell."

\section{CASE OF TUMOUR OF THE SPINAL DURA MATER.}

By WILLIAM B. RANSOM, M.A., M.D., M.R.C.P. Physician to the General Hospital, Nottingham.

WITH OPERATION BY

JOSEPH THOMPSON, M.R.C.S., Surgeon to the Hospital.

THE excellent results obtained five years ago by $\mathrm{Mr}$. Victor Horsley in the case of tumour of the spinal cord, diagnosed by Dr. Gowers, have not been always followed by equal success in other cases in the hands of others. It is therefore of importance that all instances of attempted operation for tumour in the spinal canal, whether successful or not, should be recorded and the diagnostic and therapeutic difficulties detailed

The following is the second such case in which it has fallen to my lot to invite the help of the surgeon, ${ }^{1}$ and in each the diagnosis was justified, though the final therapeutic result was in each, from special causes, disappointing.

In the former the tumour, an extradural echinococcus cyst, was found post mortem under the vertebra next above those removed, the extreme stoutness of the patient having prevented accurate counting of the spines.

In the following case the tumour, after a long operation, was found and removed, but the patient succumbed two days later. As will be seen from the report of the microscopical investigation, even if a satisfactory recovery from the operation had taken place, the nature of the growth was such that a recurrence was unavoidable.

Medical History. - C. R., aged 50, stationary engine driver, first saw me in the out-patient room on May 12th, 1893. He then complained of pain in the epigastrium and just above the pubes, and of passing blood in his water. There were no other signs of bladder or renal disease, and it was ultimately found that the suprapubic pain and hæmaturia were due to turpentine administered to him by a doctor for the stomach pain. This,
however, he did not mention for three weeks, and the difficulties of diagnosis were thereby increased.

The note of May 12th runs: "Ill two months, off work a week. Pain began in epigastrium and lower dorsal spine. It began gradually, and has been always of a dull aching character. Not made worse by breakfast or dinner, but is worse after tea, when he has some wind. The pain is worse when at work. During the last fortnight it has spread down to the pubes. He has also some trembling in the legs, but seems rather nervous and shaky all over. Has lost two stones in weight in the last two months. There is no physical sign of disease of the heart, lungs, stomach, or liver. The urine is normal, and no stone is found by the sound in the bladder. His onl previous illness is rheumatic fever nine years ago vo history of syphilis or alcoholism

It mag. No to It may sufice to say that the hæmaturia and suprapubic pain disappeared after the first visit, the reason being later made clear when the history of the turpentine was revealed. Tine epigastric pain, however, did not diminish, though it was never very severe, and he continued to complain of pain in the lower half of the dorsal spine. On further inquiry he also admitted a slight feeling of tightness in the waist. During May the trembling and weakness of the legs, which were at first not conspicuous, steadily increased, so that he had to take to a stick in walking. They also became stiff, and there were some aching pains in the front of the thighs and the calves. Whereas on May lath he could walk well, on June 2nd he walked very stifly, and complained of numbness on the front of the thighs. On this day the knee-jerks were exaggerated, but there wa ${ }^{\circ} \mathrm{n}$ ankle clonus. June 6th. Double ankle clonus, slight tactile anæsthesia on front of thighs; walks with difficulty, dragging toes on ground. June 27th. Admitted into hospital ; cannot walk, and can only
stand with support. During this time the patient had slept and eaten 1 See Ransom and Anderson, BRITISH Medical JouRnal, November 28 th, 1891 . well, and remained stout, although he said he had lost flesh. The urine and sphincters have been norma.

On July 2 nd the following note was taken

"Patient is a stout muscular man of healthy aspect. He complains of pain in the lower part of the epigastric region and a tight feeling spreading from this place round to the back, about the level of the eleventh rib. He has also pain in the back on either side of the spine about the level of the three last dorsal vertebro. All his pains are dull and not accurately defined. There are also some crampy pains in the front of
the thighs if he moves. He has a sensation of numbness on the front of the legs and thighs, more marked on the right.

"Motor power is normal in the eyes, face, arms, trunk, and sphincters, but the legs show great loss. He can just stand for a moment by himself, but cannot walk with

the left leg than the right.

"Senten is norma on the head and arms. There is an area on the front of each leg, stretching from Poupart's ligament downwards as a. narrowing cone to the lower third of the shin, on which sensibility is lost to touch, pain, and heat. On the left leg the anæsthesia is incomplete, but on the right it becomes almost complete at Poupart's ligament plete, but on the right in becomes on both soles and dorsal surfaces of the feet, on the back of the legs, thighs, and in the perineum sensation to touch and pain are normal, though heat and cold are sometimes con* fused.

"On the back of the trunk sensation seems fair, but there are a few On the back of the trunk sensation seems dorsal spines where a light points on either side of the eighth and ninth dorsal spines where a light. touch is sometimes missed. There are similar patches of very faint anæsthesia in front near the anterior ends of the eighth and ninth ribs. On the abdomen sensation is normal. Just above the slightly anæsthetic spots behind there is a

\section{tenderness is absent.}

"The knee-jerks are increased, more especially the right, and there is double ankle clonus. The plantar, cremasteric, and abdominal reflexes

are not obtained. cept that he has occasionally some flatulence after tea."
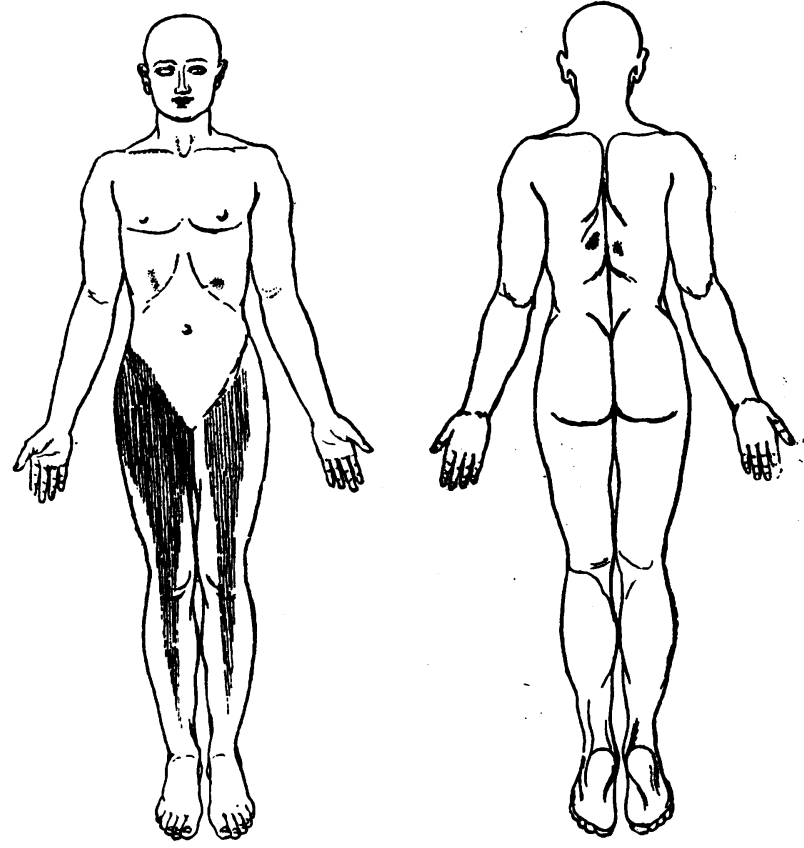

Fig. 1.-C. R. Aleas of anæsthesia. The dotted patches on the trunk indicate the spots of very slight and variable tactile anæsthesia. The deeper shading on the right leg indicates the greater intensity of the anæsthesia on that leg.

During the next fortnight, in spite of the administration of potassic mercuric iodide, the condition of the patient did not improve.

The note on July 16th says "he cannot lift either leg off the bed although the toes of both can be moved. There has been no distinct girdle sensation, and the pain in the epigastric region and back has been ess intense and ill defined.

"The sensory loss is slightly variable and is not increased. Painful impressions are felt on the left leg, but on the front of the right he often misses a prick or pinch. Pulling the hairs in the right groin may be felt on one day and not on another.

"On the trunk the disturbance of sensation is still more uncertain The patient's fat makes counting the spines difficult, but in an area of skin a square inch in size about the level of the eighth and ninth spines he usually misses a light touch on either side of the vertebral column. Occasionally on the left there seemed to be slight dulling of sensibility for an inch higher, but there is no zone of hyperæsthesia. On the front of the body there is a point about three inches below the nipple and just internal to it where he sometimes fails to detect a light touch, but this spot of partial anæsthesia cannot always be demonstrated. Otherwise 